\title{
THE
}

\section{Comparison of spin anisotropy and exchange alternation}

Jill C. Bonner

University of Rhode Island

Handrik W. J. Blöte

University of Rhode Island

J. D. Johnson

Follow this and additional works at: https://digitalcommons.uri.edu/phys_facpubs

Terms of Use

All rights reserved under copyright.

\section{Citation/Publisher Attribution}

Bonner, J. C., Blöte, H. W. J., \& Johnson, J. D. (1979). Comparison of spin anisotropy and exchange alternation. J. Appl. Phys., 50, 7379-7381. doi: 10.1063/1.326951

Available at: https://doi.org/10.1063/1.326951

This Article is brought to you for free and open access by the Physics at DigitalCommons@URI. It has been accepted for inclusion in Physics Faculty Publications by an authorized administrator of DigitalCommons@URI. For more information, please contact digitalcommons-group@uri.edu. 


\section{A|P| $\left.\right|_{\text {Applied Physics }} ^{\substack{\text { Journal of } \\ \text { Ans }}}$}

\section{Comparison of spin anisotropy and exchange alternation}

Jill C. Bonner, Hendrik W. J. Blöte, and J. D. Johnson

Citation: Journal of Applied Physics 50, 7379 (1979); doi: 10.1063/1.326951

View online: http://dx.doi.org/10.1063/1.326951

View Table of Contents: http://scitation.aip.org/content/aip/journal/jap/50/B11?ver=pdfcov

Published by the AIP Publishing

\section{Articles you may be interested in}

Elementary excitations and thermodynamics of zig-zag spin ladders with alternating nearest-neighbor exchange interactions

Low Temp. Phys. 35, 455 (2009); 10.1063/1.3151992

Effects of crystal texture on exchange anisotropy in $\mathrm{NiO}$ spin valves

J. Appl. Phys. 81, 5298 (1997); 10.1063/1.364948

Spin diffusion in classical Heisenberg magnets with uniform, alternating, and random exchange J. Appl. Phys. 75, 6751 (1994); 10.1063/1.356842

Studies in Exchange and Anisotropy Driven Amorphousness in Finite Size Spin Systems

AIP Conf. Proc. 18, 615 (1974); 10.1063/1.3141782

Biquadratic Exchange and Spin Exchange

AIP Conf. Proc. 10, 558 (1973); 10.1063/1.2946963

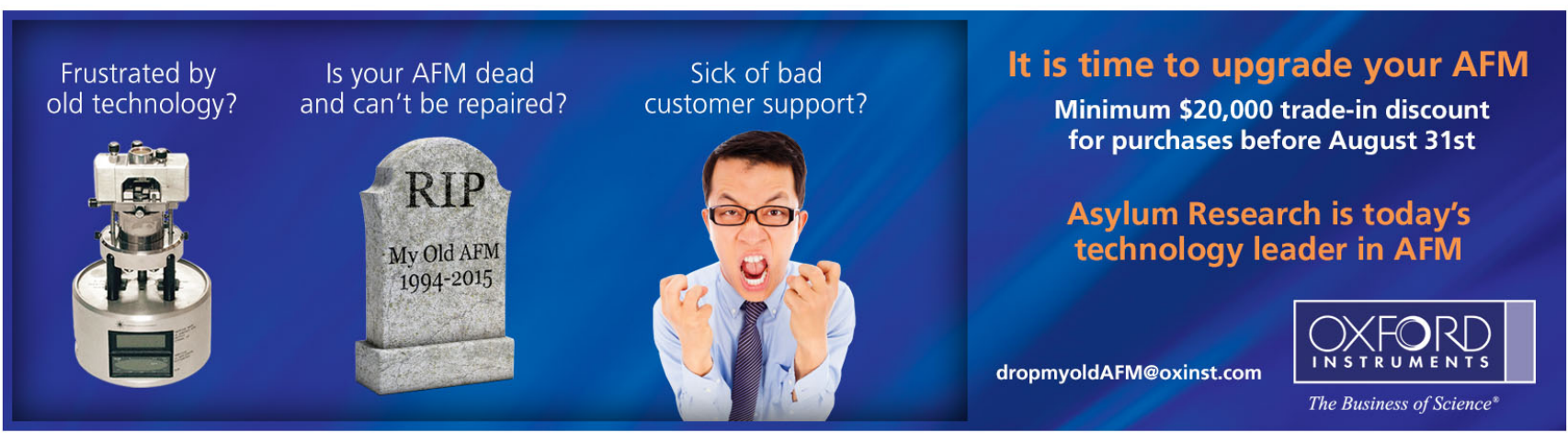




\title{
Comparison of spin anisotropy and exchange alternation
}

\author{
Jill C. Bonner ${ }^{a, b}, c$ and Hendrik W. J. Blöte ${ }^{a}$ \\ University of Rhode Island, Kingston, R.I. 02881 \\ J. D. Johnson ${ }^{d}$ \\ Los Alamos Scientific Laboratory, Los Alamos, N.M. 87544 \\ Quasi-1-D magnetic systems with on the one hand an Ising-Heisenberg type spin anisotropy and on the \\ other hand an alternating (dimerized) character have many interesting features in common and a few \\ interesting differences in their phase behavior and general magnetic properties. This report reviews results \\ rather scattered in the literature in addition to presenting new results. These rather complex quantum \\ models present a theoretical challenge. It is also hoped that this work will be helpful to magnetochemists \\ interested in identifying the underlying magnetic character of their systems, and to experimentalists in \\ general.
}

PACS numbers: $75.10 . \mathrm{Jm}, 75.40 . \mathrm{Fa}, 75.30 . \mathrm{Kz}, 75.30 . \mathrm{Ds}$

\section{INTRODUCTION}

Quasi-one-dimensional systems have the virtue that various types of magnetic behavior can be investigated rather thoroughly, both theoretically and experimentally. From time to time it has been noted that one-dimensional spin models with anisotropy of the Ising-Heisenberg type have qualitative features in common with one-dimensional models which one isotropic in spin-space, i.e. Heisenberg, but with exchange constants $J_{1}$ and $J_{2}$ alternating in magnitude. There seems at present no clear theoretical arguments to explain this similarity: the two systems do not, for example, belong in the same universality class. In fact, as we shall see below, the resemblance is strong, but not quite complete. Certain aspects of the phase behavior of the weakly interacting spin systems do show some interesting differences. Homologous series of compounds with a uniform character are currently under scrutiny by coordination chemists, who study the variation of $\mathrm{J}$ with bond length, bond angle and, perhaps, other factors. These empirical studies can then be used to check the reliability of current theories of superexchange. Very recently, families of alternating (also called dimerized) spin systems have been discovered, which may also be used for this purpose. The problem for magneto-chemists is which model to use; spin anisotropic or alternating. These considerations, and also current interest in the spin-Peierls phenomenon, ${ }^{1}$ have motivated this study.

\section{SPECTRAL EXCITATIONS}

The Hamiltonians for the two types of system may be written (a11 systems discussed have spin $S=1 / 2$ ):

$\frac{\text { Spin Anisotropy }}{(S A)}: \quad H=2 J_{i=1}^{N}\left\{S_{i}{ }^{z} S_{i+1}{ }^{z}+\gamma\left(S_{i}{ }^{x} S_{i+1} x+S_{i}{ }^{y} S_{i+1}{ }^{y}\right)\right\}$

where the anisotropy parameter, $\gamma$, varies between 0 (Ising model) and 1 (Heisenberg mode1).

$\frac{\text { Alternation }}{(\text { AIt.) }}: \quad H=2 J_{i=1}^{N / 2}\left\{\vec{s}_{2 i-1} \cdot \vec{s}_{2 i}+\alpha \vec{s}_{2 i} \cdot \vec{s}_{2 i+1}\right\}$,

where the alternation parameter, $\alpha$, varies between 0 (1imit of isolated dimer spin pairs) and 1 (uniform mode1). We consider $\mathrm{J}>0$, antiferromagnetic. Hamiltonian (2) may also be written in terms of exchange constants $\mathrm{J}_{1}$ and $\mathrm{J}_{2}$, where $\mathrm{J}_{1} \equiv \mathrm{J}$ and $\mathrm{J}_{2} \equiv \alpha \mathrm{J}\left(\mathrm{J}_{2}<\mathrm{J}_{1}\right)$. In connection with spin-Peierls theory ${ }^{2}$ t is often convenient to define a dimerization parameter, $\delta=(1-\alpha)$ / $(1+\alpha)=\left(\mathrm{J}_{1}-\mathrm{J}_{2}\right) /\left(\mathrm{J}_{1}+\mathrm{J}_{2}\right)$.
An analytic solution is available for the lowestlying excitations of Hamiltonian (1) ${ }^{2}$, but at present no non-trivial exact analytic results are available for any property of Hamiltonian (2). It would therefore be valuable to begin by reviewing a related pair of Hamiltonians, for the Ising-XY linear chain and the alternating $X Y$ chain, both of which may be solved exactly via a standard transformation from spin to pseudo-fermion operators. The Hamiltonians are:

$\mathrm{SA}:$

$$
\begin{aligned}
& \mathrm{H}=2 \mathrm{~J}_{i} \sum_{i=1}^{N}\left\{\mathrm{~s}_{i}^{\mathrm{x}} \mathrm{s}_{i+1}^{\mathrm{x}}+\gamma \mathrm{s}_{i}^{\mathrm{y}} \mathrm{s}_{i+1}^{\mathrm{y}}\right\} \\
& H=2 J \underset{i=1}{N / 2}\left\{S_{2 i-1}^{x} S_{2 i}^{x}+s_{2 i-1}^{y} S_{2 i}^{y}\right. \\
& \left.+\alpha\left(s_{2 i}^{x} s_{2 i+1}^{x}+s_{2 i}^{y} s_{2 i+1}^{y}\right)\right\} \text {. }
\end{aligned}
$$

Both models are variants on the $1-D$ uniform XY model, first solved by Lieb et al. and Katsura ${ }^{3}$.

A comparative discussion is facilitated by considering a more general Hamiltonian, containing (3) and (4) as particular cases, studied by Dubois and Carton and Perk and Cape1.4 The exact dispersion relations are:

$E_{1}(q) \sim \frac{2(1+\gamma \alpha)}{(1+\gamma)(1+\alpha)}\left[\cos ^{2} q+\left(\frac{1-\gamma \alpha}{1+\gamma \alpha}\right)^{2} \sin ^{2} q\right]^{\frac{2}{2}}$
$E_{2}(q) \sim \frac{2(\gamma+\alpha)}{(1+\gamma)(1+\alpha)}\left[\cos ^{2} q+\left(\frac{\gamma-\alpha}{\gamma+\alpha}\right)^{2} \sin ^{2} q\right]^{\frac{1}{2}}$.

Note first of all the complete equivalence of $\gamma$ and $\alpha$ in (5) and (6). If either $\gamma=1$ (isotropic case) or $\alpha=1$ (uniform case), (5) and (6) become degenerate as, say

$$
\varepsilon_{1,2}(q) \sim\left[\cos ^{2} q+\left(\frac{1-\gamma}{1+\gamma}\right)^{2} \sin ^{2} q\right]^{\frac{1}{2}} \text {. }
$$

The excitations are gapless when $\alpha=\gamma=1$, otherwise the energy gap, $\Delta E \sim(1-\gamma)$ or $(1-\alpha)$. It is very interesting that there is another case when branch (6) of the excitations is gapless, namely when $\gamma=\alpha$. In some sense the anisotropy and alternation compensate so as to give quasi-uniform-Heisenberg behavior. This implies that an anisotropic XY spin-Peierls system should show unusual behavior, and possibly an Ising-Heisenberg spinPeierls system also.

Let us now compare the Heisenberg-like systems $[(1),(2)]$ with the XY-like systems $[(3),(4)]$. There is a qualitative similarity in that models (1) and (2) show a spectral excitation gap vanishing only in the limits $\gamma=1$ or $\alpha=1,5,6$ respectively. However, both gaps now have very ditterent tunctional forms. In Fig. I we show a sequence of exact finite chain anisotropy energy gaps for systems of up to 12 spins, together with an extrapolated, numerical, $\mathrm{N} \rightarrow \infty$ estimate (indicated by crosses). An exact analytic result is available ${ }^{2}$. The gap rises 


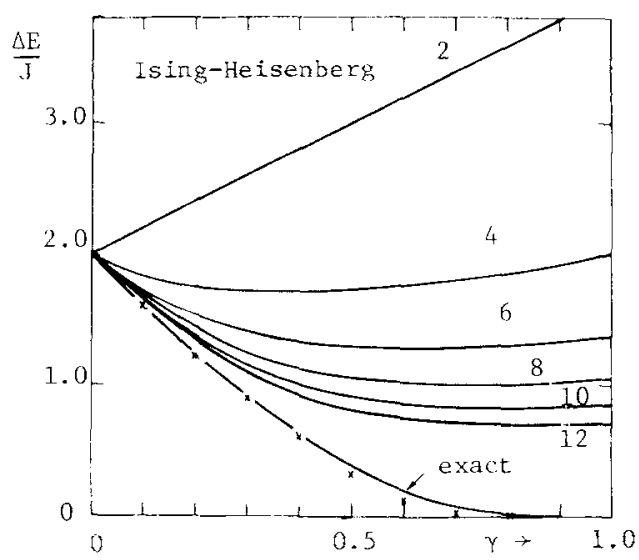

Fig. 1 Ising-Heisenberg energy gaps as function of $\gamma$, showing finite $N$ results, the exact result, and extrapolations (crosses).

exponentially slowly away from the Heisenberg limit [as $\left.\exp \left(-\mathrm{A}[\mathrm{I}-\gamma]^{-2}\right)\right]$.

The agreement with numerical extrapolations is really quite good 7,8 over the whole $\gamma$ range. This is important in connection with Fig. 2, where we show alternation energy gaps. Since no analytic solution exists for this problem, we will rely on numerical extrapolations. Again, a sequence of exact finite chain alternation gaps for systems of up to 12 spins is shown, together with $\mathrm{N} \rightarrow \infty$ extrapolations (dashed curve). The gap apparently vanishes only in the uniform (Heisenberg) limit, but the functional form is quite different from the spin anisotropy case. A theoretical approach by Cross and Fisher ${ }^{9}$ based on the Luttinger-Luther-Pesche1 continuum lattice model predicts that $\Delta E \backsim \delta^{2 / 3}$, consistent with a zero temperature RG approach 10 and also (qualitatively) with the extrapolations.

The specific heat and susceptibility of IsingHeisenberg chains has been investigated both numerically 11 and analytically 7 , and agreement is good. Both specific heat and parallel susceptibility have typical rounded maxima, and vanish exponentially as $\mathrm{T} \rightarrow 0$ on account of the energy gap. For the alternating model, numerical calculations of Duffy and Barr ${ }^{12}$ have been extended by considering longer chains (up to 12 spins) so that reliable extrapolations may be made closer to the uniform limit. Detailed results for the susceptibility for a wide range of a have been presented 13 and new results for the specific heat and zero-point magnetization in a field are available. Again, specific heat and susceptibility have rounded maxima, and vanish exponentially as $\mathrm{T}+0$ on account of the energy gap.

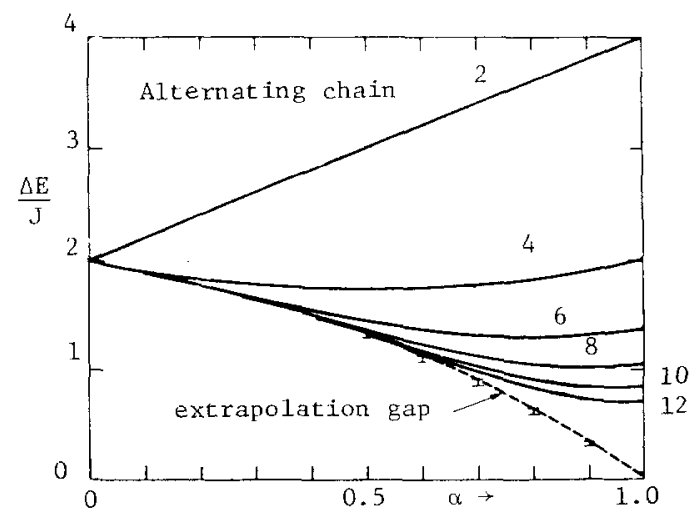

Fig. 2 Alternating energy gaps as function of $\alpha$, showing finite $\mathrm{N}$ results, extrapolations and error bars.
The important difference between the two types of systems lies in the susceptibility. Since the alternating chain remains isotropic in spin space, there is no distinction between parallel and perpendicular susceptibility. The susceptibility for both powder and single crystals always goes to zero at $\mathrm{T}=0$. For the IsingHeisenberg model, only the parallel susceptibility vanishes, the perpendicular susceptibility is finite and non-zero. Closely related is the low temperature magnetization as a function of field. For powder Ising-Heisenberg samples, the magnetization is always non-zero for $\mathrm{H}>0$, whereas for powder samples of alternating chains, the magnetization remains zero until a threshold field is reached, when it rises rather rapidly. This feature has been used to identify copper bromide imidazole as an alternating system, whereas chenically related sister compounds apparently behave like uniform chains. 14

\section{CRITICAL BEHAVIOR}

The simple antiferromagnet (AFM) has a single critical field (at $\mathrm{T}=0$ ) above which AFM ordering is destroyed by the field. For both the spin anisotropic and alternating $1-D$ models there are two critical fields, lower and upper, $\mathrm{H}_{\mathrm{cl}}$ and $\mathrm{H}_{\mathrm{c} 2}$, respectively. For both models, $\mathrm{H}_{\mathrm{c} 2}$ is given exactly by simple spin-wave theory. Again, for both models, $\mathrm{H}_{\mathrm{cl}}$ is a direct measure of $\Delta \mathrm{E}$, the energy gap, since $\mathrm{H}_{c I}$ is the field at which a (magnetic) component of the excited states above the gap crosses the non-magnetic ground state(s) on account of the Zeeman term. For the XY-1ike models, of course, both $\mathrm{H}_{\mathrm{cl}}$ and $\mathrm{H}_{\mathrm{c} 2}$ are known exactly. For Heisenberglike models we have:-

SA:

$$
\mathrm{H}_{\mathrm{c} 2}=2 \mathrm{~J}(\mathrm{I}+\gamma) \text {. }
$$

$\mathrm{H}_{\mathrm{cl}}$ can be found from the graph of Fig. 1, where the expression plotted can be obtained from ref. 2, equations $(161,162)$, or Yang and Yang 15 , equation (8).

Alt: $\quad \mathrm{H}_{\mathrm{c} 2}=2 \mathrm{~J}_{1}(1+\alpha)$.

$\mathrm{H}_{\mathrm{cl}}$ can be found approximately from the graph of Fig. 2 . There is also a third-order perturbation calculation of Harris 16 which gives

$$
\mathrm{H}_{\mathrm{Cl}} / J_{1} \approx \Delta \mathrm{E} / \mathrm{J}_{1} \simeq 2-\alpha-\frac{3}{4} \alpha^{2}+\frac{1}{16} \alpha^{3}+\ldots
$$

This expression converges well from the dimer limit out to $\alpha=0.5$ and agrees with the numerical extrapolations in this alternation range. Note the isom orphism between $\gamma$ and $\alpha$ in (8) and (9). At $\mathrm{T}=0$, critical singularities occur in $X_{(T=0)}$, but space prevents a discussion here.

For both Ising-Heisenberg and alternating chains, the behavior of the thermodynamic properties, e.g. the specific heat, is very similar. For both models we have the same functional forms for $\mathrm{T} \rightarrow 0$ :

$$
\begin{aligned}
& 0 \leq \mathrm{H}<\mathrm{H}_{\mathrm{Cl}}: \quad \mathrm{C}_{\mathrm{H}} \backsim \mathrm{T}^{-3 / 2} \mathrm{e}^{-\Delta \mathrm{E} / \mathrm{kT}} \\
& \mathrm{H}=\mathrm{H}_{\mathrm{c} 1} \text { and } \mathrm{H}=\mathrm{H}_{\mathrm{c} 2}: \quad \mathrm{C}_{\mathrm{H}} \curvearrowright \mathrm{A}_{1,2} \mathrm{~T}^{1 / 2} \\
& \mathrm{H}_{\mathrm{Cl}}<\mathrm{H}<\mathrm{H}_{\mathrm{C} 2}: \quad \mathrm{C}_{\mathrm{H}} \curvearrowright \mathrm{B}(\mathrm{H}) \mathrm{T} .
\end{aligned}
$$

Exact results for the Ising-Heisenberg model expanded in $\gamma$ are: $A_{1}=0.114 ; \quad A_{2} \simeq 0.229$. (13) $\sqrt{8 y}$

$$
\sqrt{2 \gamma}
$$

For the alternating model, no such exact results are available but spin-wave theory and detailed experiment 17 confirm the correctness of the functional forms $(10,11$, 12). Note that for both models, the behavior resembles that expected for a uniform $A F M$ at $T=0$ in the range $\mathrm{H}<\mathrm{H}<\mathrm{H}_{2}$; whereas at $\mathrm{H}=\mathrm{H}$, the models resemble a zero-field ferromagnet. In both models there is asymmetry i.e. $A_{1}<A_{2}$.

A zero temperature quantum RG method has been formulated where the quantum chain is divided into exactly solvable blocks of $\mathrm{N}_{\mathrm{s}}$ spins, and the inter-block coup1ing is written in terms of a truncated block vector basis 6,10 . For alternating chains with $\mathrm{N}_{\mathrm{S}}=3$, the $\mathrm{RG}$ iteration process yields two fixed points, $\alpha^{*}=1$ 


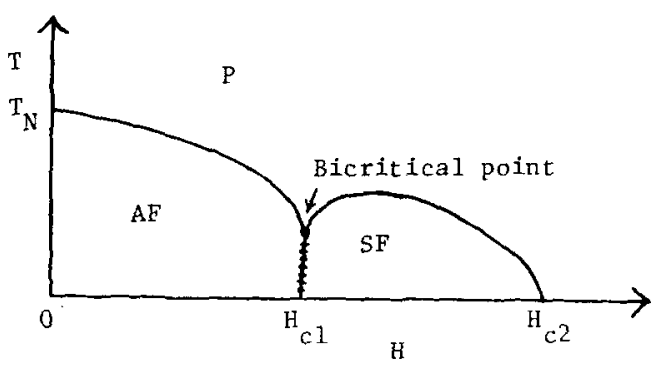

Fig. 3 Quasi-1-D spin-flop AFI phase diagram, showing $A F$ ordered region, spin-flop region (SF) and bicritical point.

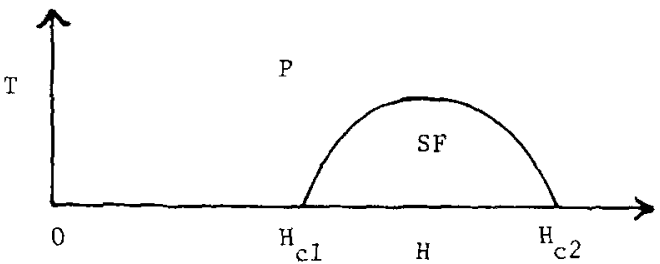

Fig. 4 Quasi-1-D alternating AFM phase diagram, showing spin-flop (SF) ordered region.

(unstable) and $\alpha^{*}=0(\infty)$ (stable). This result has the important physical implication that the system with non-zero alternation, i.e. $\alpha<1$, will renormalize into a dimer system, characterized by a singlet-triplet energy gap. This implies that the excitation gap vanishes only in the uniform limit, $\alpha=16$. Repeating the calculations for a uniform Ising-Heisenberg system ${ }^{19}$ strengthens this interpretation. Fixed points are found at $\gamma^{*}=1$ (unstable) and $\gamma^{*}=0, \infty$ (stable). This agrees with exact results ${ }^{2}$. If the RG calculations are repeated for the corresponding $X Y$-type models, similar fixed point behavior appears, again in agreement with the exact results. It is interesting that the RG method gives equivalent recursion relations for both cases (3) and (4), apparently reflecting the equivalence of the two exact solutions noted above.

When the 1-D systems weakly interact, cooperative ordering occurs for $\mathrm{T}>0$. Since none of our systems is now exactly solvable, the following discussion relies on spin-wave theory, mean-fleld theory and experiment. The phase diagram for a quasi-1-D Ising-Heisenberg AFM, which is, of course, a spin-flop system is sketched in Fig. 3. The usual three phases appear, AFM, spin-flopped and paramagnetic. The corresponding phase-diagram for a quasi-1-D alternating AFM is sketched in Fig. 4. The surprising feature is the complete absence of the AFM phase. There is no phase transition of any type in zero field: cooperative ordering of a spin-flop type occurs only between $H_{C l}$ and $H_{2}$. There is likewise no trace of a first order anti/floptransition line: The phase boundary is second order. Such behavior typifies copper nitrate, $\mathrm{Cu}\left(\mathrm{NO}_{3}\right)_{2} \cdot 2.5 \mathrm{H}_{2} \mathrm{O}$, which has been very extensively studied, both experimentally and theoreticalIy 17,18. The presence of the AFM ordered phase in Fig. 3 is related to the existence of long-range-order (LRO) at $T=0$ of the Ising-Heisenberg chain. The alternating chain has a non-degenerate singlet ground state; hence no LRO at $\mathrm{T}=0$, and therefore no transition will appear for $T>0$ when the chains interact.

\section{MAGNETIC COOLING}

Quas1-1-D Ising-Heisenberg and alternating systems may show a striking difference in their phase behavior. but they show strong similarities in their cooling behavior, 1.e. isentropes as a function of field and temperature. This is because magnetic cooling is intimately related to the entropy, on which the effects of a second order or continuous transition are rather minimal. Fig. 5 shows the results of an analytic calculation on an ideal Ising-Heisenberg chain with $\gamma=0.2 .20$ Note

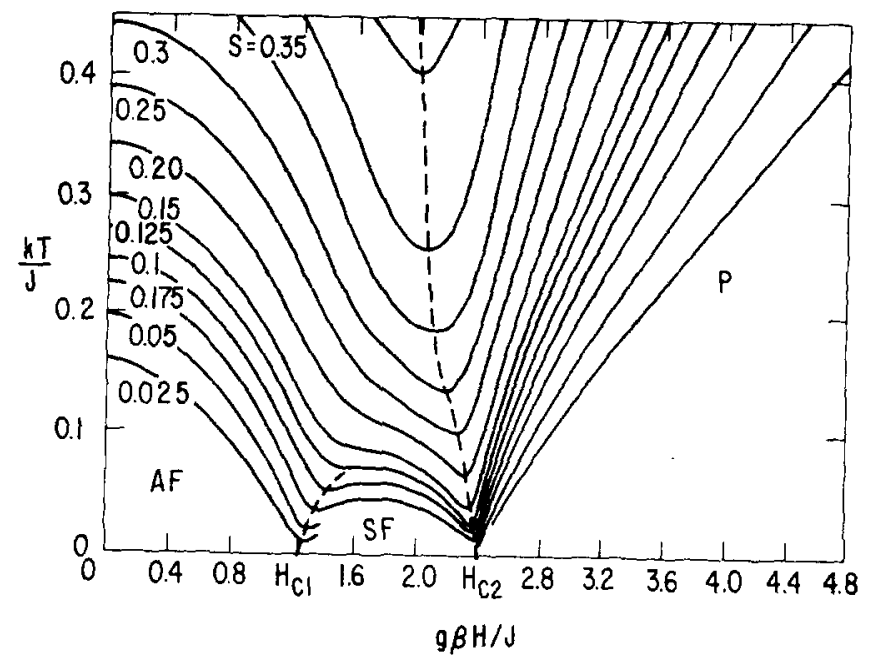

Fig. 5 Exact cooling isentropes for Ising-Heisenberg chain for $\gamma=0.2$.

that four different types of cooling behavior, including both adiabatic magnetization and adiabatic demagnetization, are observed in this system. Entropy maxima and hence isentrope minima appear in the vicinity of $\mathrm{H}_{\mathrm{cl}}$ and $\mathrm{H}_{\mathrm{c} 2}$. For the alternating chain system copper nitrate, the cooling curves (theoretical and experimental) are remarkably similar to the Ising-Heisenberg case. 17

\section{REFERENCES}

(a) Supported by NSF Grant \#DMR77-24136.

(b) Supported by NATO.

(c) Fellow of the Bunting Institute, Radcliffe College.

(d) Supported by DOE.

1. I.S. Jacobs et al., Phys. Rev. B 14, 3036 (1976).

2. J. des Cloizeaux and M. Gaudin, J. Math. Phys. 7 1384 (1966). For corrected excitations, see e.g. J.D. Johnson and B.M. McCoy, Phys. Rev. A 6, 1613 (1972).

3. E. Lieb, T. Schultz and D. Mattis, Ann. Phys. 16, 407 (1961): S. Katsura, Phys. Rev. 127, 1508 (1962)

4. J.Y. Dubois and J.P. Carton, J. Phys. (Paris) 35. 371 (1974): J.H.H. Perk et al., Physica 81A, $3 \frac{319}{19}$ (1975).

5. J.C. Bonner, H.W.J. Blbte and J.D. Johnson, J. Phys. (Paris) 39, G6-710 (1978)

6. J.N. Fields, Phys. Rev. B 19, 2637 (1979)

7. See J.D. Johnson, Phys. Rev. A 9, 1743 (1974).

8. J.C. Bonner and H.W.J. Blote, unpublished work.

9. M.C. Cross and D.S. Fisher, Phys. Rev. B 19, 402 (1979).

10. J.N. Fields, J.C. Bonner and H.W.J. Bldte, J. Appl. Phys. 50, 1807 (1979): H.W.J. B18te, J.C. Bonner and J.N. Fields, paper to be presented at ICM 79 Munich.

11. J.C. Bonner and M.E.Fisher, Phys. Rev. 135, A640 (1964).

12. W. Duffy, Jr. and K.P. Barr, Phys. Rev. 165, 647 (1967).

13. J.C. Bonner, H.W.J. Bl8te, J.W. Bray and I.S. Jacobs, J. App1. Phys. 50, 1810 (1979).

14. J.J. Smit, L.J. de Jongh, J.A.C. van ooijen, J. Reedijk and J.C. Bonner, Physica (in press).

15. C.N. Yang and C.P. Yang, Phys. Rev. 151, 258 (1966).

16. A.B. Harris, Phys. Rev, 157, 295 (1967).

17. K.M. Diederix, H.W.J. Blbte, J.P. Groen, T.o.Klaasen, and N.J. Poulis, Phys. Rev. B 19, 420 (1979).

18. J.C. Bonner et a1., Proc. 12th Int. Conf. on Low Temp. Phys., Kyoto, Japan, p. 691 (1970).

19. J.N. Fields, private communication.

20. J.C. Bonner and J.D. Johnson, Physica 86-88B, 653 (1977). 